\section{¿Puede el ejercicio físico per se disminuir el peso corporal en sujetos con sobrepeso/obesidad?}

\author{
CARLOS BURGOS ${ }^{1, \mathrm{a}, \mathrm{b}}$, CARLOS HENRÍQUEZ-OLGUÍN ${ }^{1, \mathrm{a}, \mathrm{b}}$, \\ RODRIGO RAMÍREZ-CAMPILLO ${ }^{1,2, a, c}$, SANDRA MAHECHA MATSUDO ${ }^{1,3, d}$, \\ HUGO CERDA-KOHLER ${ }^{1, a, b}$
}

\section{Exercise as a tool to reduce body weight}

The prevalence of overweight and obesity is increasing, creating a public health problem. The loss of approximately $10 \%$ of body weight is recommended to reduce the risk of mortality associated with metabolic diseases and to increase the quality of life in adults with overweight or obesity. Non-pharmacological strategies used for weight management are caloric restriction and physical exercise. Nevertheless, the independent effect of physical exercise to decrease body weight is unclear, and could be responsible for only $20 \%$ of the weight loss when healthy lifestyles are prescribed. However, exercise has other benefits for health, independent of its weight reducing effect. In fact, physical inactivity is responsible for twice the deaths caused by obesity. The aim of this review is to discuss the importance of physical exercise in the reduction of body weight in subjects with overweight or obesity.

(Rev Med Chile 2017; 145: 765-774)

Key words: Exercise; Obesity; Overweight; Weight Loss.
'Laboratorio de Ciencias del Ejercicio, Clínica MEDS. Santiago, Chile.

${ }^{2}$ Departamento de Ciencias de la Actividad Física, Universidad de Los Lagos. Osorno, Chile. ${ }^{3}$ Facultad de Medicina, Universidad Mayor. Santiago, Chile

aProfesor de Educación Física. ${ }^{\mathrm{b}}$ Magíster en Ciencias Fisológicas. cPhD en Ciencias del Ejercicio. dMagíster en Salud y Ejercicio.

No hubo fuentes de apoyo financiero para la realización de este manuscrito.

Recibido el 11 de marzo de 2016 aceptado el 7 de septiembre de 2017.

Correspondencia a: Carlos Burgos Jara Dirección: Isabel la Católica 3740. Las Condes burgoschile@gmail.com

\section{I} a prevalencia de sobrepeso y obesidad se ha incrementado dramáticamente en todo el mundo, convirtiéndose en un problema de salud pública que aumenta el riesgo de desarrollar enfermedades crónicas no transmisibles $(\mathrm{ECNT})^{1,2}$. Un adecuado control del peso corporal se asocia a una reducción de factores de riesgo como hipertensión arterial, diabetes mellitus tipo 2 y dislipidemia ${ }^{3}$. Diversos estudios e instituciones ligadas a la salud, como la Organización Mundial de la Salud y los National Institutes of Health han sugerido que la pérdida de aproximadamente $10 \%$ del peso corporal en adultos con sobrepeso/ obesidad puede disminuir el riesgo de mortalidad asociada a enfermedades metabólicas e incrementar su calidad de vida ${ }^{4-6}$. En general, el sobrepeso/ obesidad también está asociado a un estado inflamatorio crónico leve, el cual es una de las causas del desarrollo de resistencia a la insulina en el músculo esquelético ${ }^{7}$. Sin embargo, paradójicamente, no todos los sujetos con sobrepeso/obesidad presentan riesgos metabólicos y cardiovasculares, y se ha estimado que aproximadamente $20-30 \%$ de esta población es "metabólicamente sana" ${ }^{-10}$. No obstante, esta población presenta daño vascular ${ }^{11}$, y a largo plazo ( $\geq 10$ años) poseen mayor riesgo de contraer todas las causas y eventos de mortalidad metabólica y cardiovascular ${ }^{12}$.

Las estrategias no farmacológicas más utilizadas para el control del peso corporal son la restricción calórica y el ejercicio físico ${ }^{13-15}$. La restricción calórica es una intervención dietaria baja en calorías (reducción de aproximadamente $10-30 \%$ en relación a las necesidades diarias) pero que mantiene los requerimientos o necesidades nutricionales ${ }^{16}$. El ejercicio físico (EF) es un tipo de actividad motora planificada, estructurada y repetitiva que tiene un objetivo en particular ${ }^{17}$, sin embargo, su efecto en la disminución del peso corporal aún no está del todo claro. En este 
sentido, se ha mostrado que cuando el EF es utilizado sin una restricción calórica, los efectos en la disminución del peso corporal son modestos, en comparación a la combinación de ambas modalidades terapéuticas ${ }^{18}$. El bajo impacto que tiene el ejercicio per se en el peso corporal podría deberse a que este último es regulado por múltiples factores, lo que se muestra en la Figura 1 y que plantea la complejidad asociada al desarrollo de la obesidad.

A pesar que la disminución del peso corporal está fuertemente ligado a un proceso de gasto de energía, la etiología es compleja y aún en proceso de estudio. Además, el concepto de balance energético (e.g., balance energético = ingesta de energía-gasto de energía), si bien es un componente importante a tener en cuenta en pacientes con sobrepeso/obesidad, entendemos que es sólo una mirada simplista al problema y no entrega una solución integral (Figura 1). Un balance energético negativo generado sólo por el EF no responde a las expectativas de disminución del peso corporal, lo que se suma a los tiempos prolongados (6-12 me- ses) de espera para obtener cambios significativos de esta variable ${ }^{19}$. Así, el efecto del EF respecto a la disminución del peso corporal en esta población es ambiguo, modesto y de difícil pronóstico. Una revisión sistemática y metaanálisis de 14 estudios clínicos controlados donde se incluyeron 1.847 pacientes obesos, mostró que el EF, per se, no disminuía significativamente el peso corporal ${ }^{20}$. Otro metaanálisis de 493 estudios con pacientes obesos comparó el efecto de la restricción calórica, del EF y la combinación de estos en relación a la pérdida de peso corporal total (Figura 2) y del tejido adiposo (Figura 3). En este estudio se observó que, luego de aproximadamente 15 semanas, los tres tipos de intervenciones indujeron pérdidas de $10,7 \pm 0,5 \mathrm{~kg}, 2,9 \pm 0,4 \mathrm{~kg}$ y $11,0 \pm 0,6 \mathrm{~kg}$ en el peso corporal, con un tamaño del efecto de 5,1 $\pm 0,5$, $2,1 \pm 0,5$ y $5,5 \pm 0,7$ (restricción calórica, EF y $\mathrm{EF}+$ restricción calórica, respectivamente $)^{21}$. Los resultados sugieren que el EF, per se, podría tener un efecto aditivo de aproximadamente sólo 20\% en relación a la pérdida inicial de peso corporal

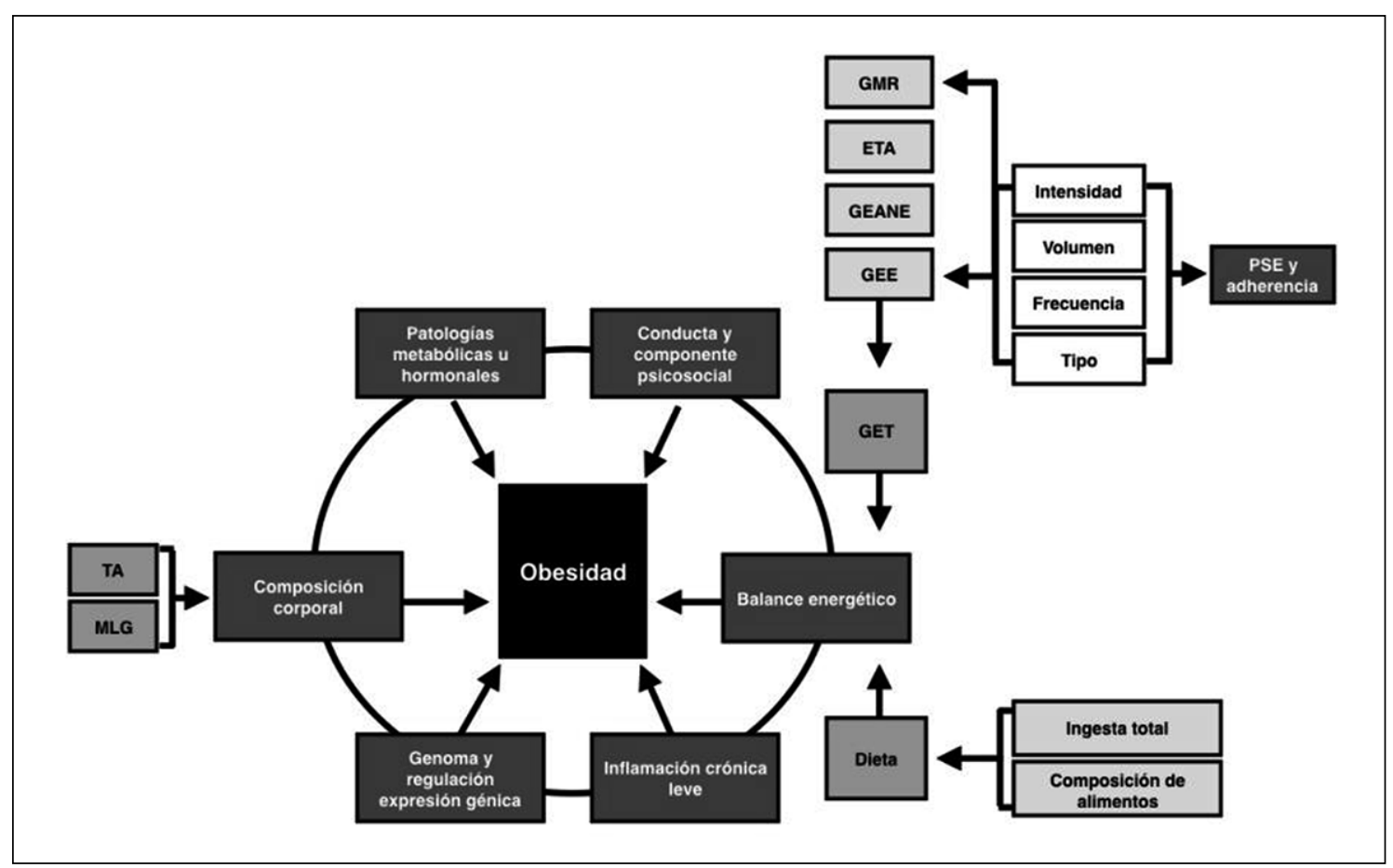

Figura 1. Representación esquemática de los múltiples factores que pueden influir en la prevención, desarrollo o tratamiento de la obesidad. TA: tejido adiposo; MLG: masa libre de grasa; GET: gasto energético total; GEE: gasto energético por ejercicio; GEANE: gasto energético por actividades que no son ejercicio; ETA: efecto térmico de los alimentos; GMR: gasto metabólico de reposo; PSE: percepción subjetiva del esfuerzo. 


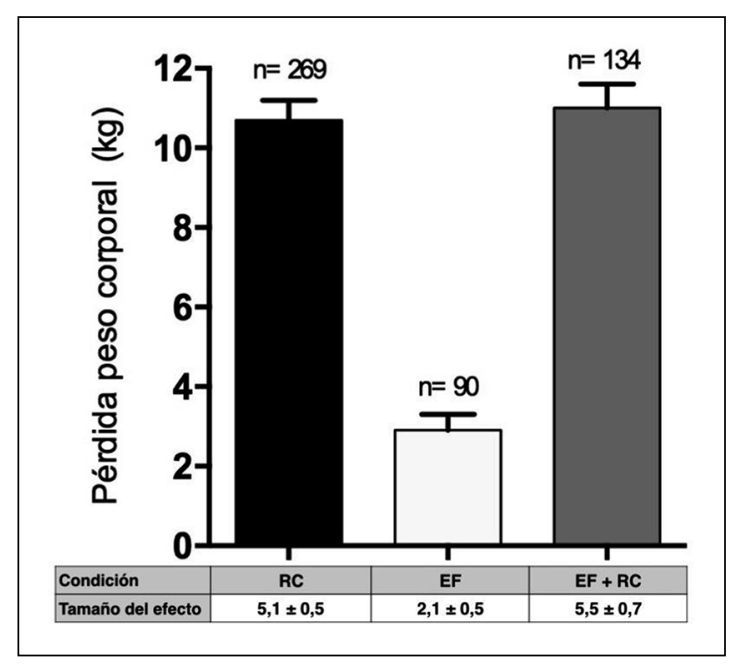

Figura 2. Pérdida de peso corporal total con diferentes estrategias no farmacológicas para el tratamiento del sobrepeso/ obesidad ${ }^{21}$. RC: restricción calórica; EF: entrenamiento físico; kg: kilogramos.

inducida por restricción calórica ${ }^{20}$. Por lo tanto, al manejo dietario debe incorporase un programas de EF para maximizar el logro de objetivos ${ }^{22}$.

\section{Aplicación de modelos de ejercicio continuo de baja a moderada intensidad y ejercicio intermitente de alta intensidad en pacientes con sobrepeso/obesidad}

\section{Ejercicio continuo}

El ejercicio continuo puede definirse como una actividad motora continua de aproximadamente 5-240 min de duración realizada a una intensidad $\leq 100 \%$ del máximo consumo de oxígeno ${ }^{23}$ y puede ser clasificado de baja, moderada o alta intensidad en función del esfuerzo aplicado y el tiempo realizado ${ }^{17}$. Este modelo de ejercicio ha sido tradicionalmente el más utilizado en el tratamiento del sobrepeso/obesidad, y ha mostrado ser efectivo en la protección cardio-metabólica, en la elevación de la capacidad de trabajo físico ${ }^{24,25}$, en el incremento de la biogénesis mitocondrial ${ }^{26}$, expresión y traslocación de los transportadores de glucosa de tipo 4 (GLUT-4) ${ }^{27,28}$, aumentos en la sensibilidad a la insulina ${ }^{29}$, en una mejor regulación de la glicemia ${ }^{30}$ y de los lípidos en sangre $^{31}$. Debido a esto, algunos consideran este

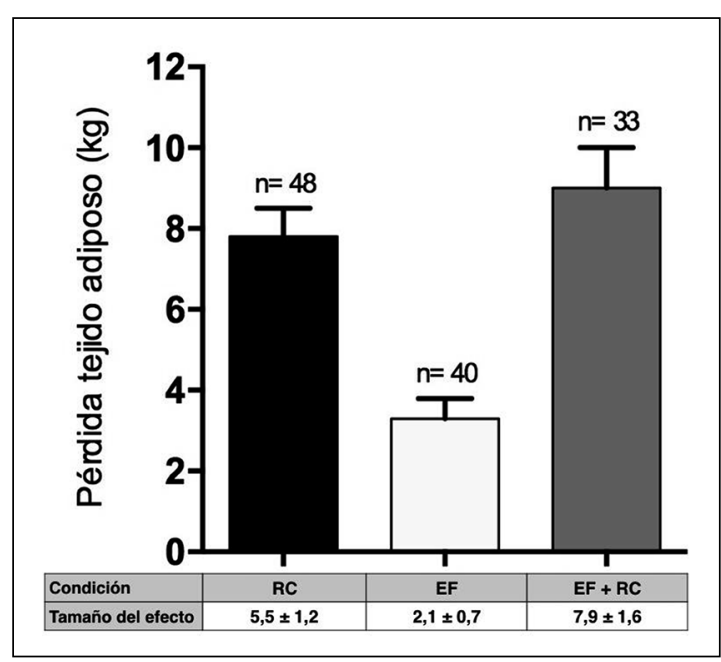

Figura 3. Pérdida de tejido adiposo total con diferentes estrategias no farmacológicas para el tratamiento del sobrepeso/obesidad ${ }^{21}$. RC: restricción calórica; EF: entrenamiento físico; kg: kilogramos.

modelo de ejercicio como el fármaco ideal en el tratamiento de diabetes mellitus tipo 2, síndrome metabólico e hipertensión arterial ${ }^{32}$. Sin embargo, la gran variedad de protocolos encontrados en la literatura dificulta la identificación de la intensidad y el volumen óptimo para inducir cambios significativos en el peso corporal en pacientes con sobrepeso/obesidad.

El ejercicio continuo necesita aproximadamente 50-60\% más de tiempo de realización en comparación con otros modelos de ejercicio ${ }^{33,34}$. No obstante, es ampliamente utilizado en la prevención primaria de enfermedades metabólicas y cardiovasculares, con una dosis de al menos 30 min de ejercicio de moderada intensidad y una frecuencia de 5 veces por semana ${ }^{17}$. Hay estudios que proponen que este modelo de entrenamiento estaría asociado a una mayor adherencia en el tiempo ${ }^{35,36}$, sin embargo, a pesar de sus beneficios sobre diferentes factores de salud, presenta un limitado impacto sobre la disminución del peso corporal, aproximadamente $2,4 \%(0,3 \%-8,4 \%)$ en un plazo de 6 a 18 meses de aplicación del $\mathrm{EF}^{37,38}$.

Un factor a considerar en la aplicación del modelo de ejercicio continuo es la relación entre la intensidad del ejercicio y la utilización de sustratos por el musculo esquelético (Figura 4). Se ha reportado que la intensidad a la cual ocurre la 


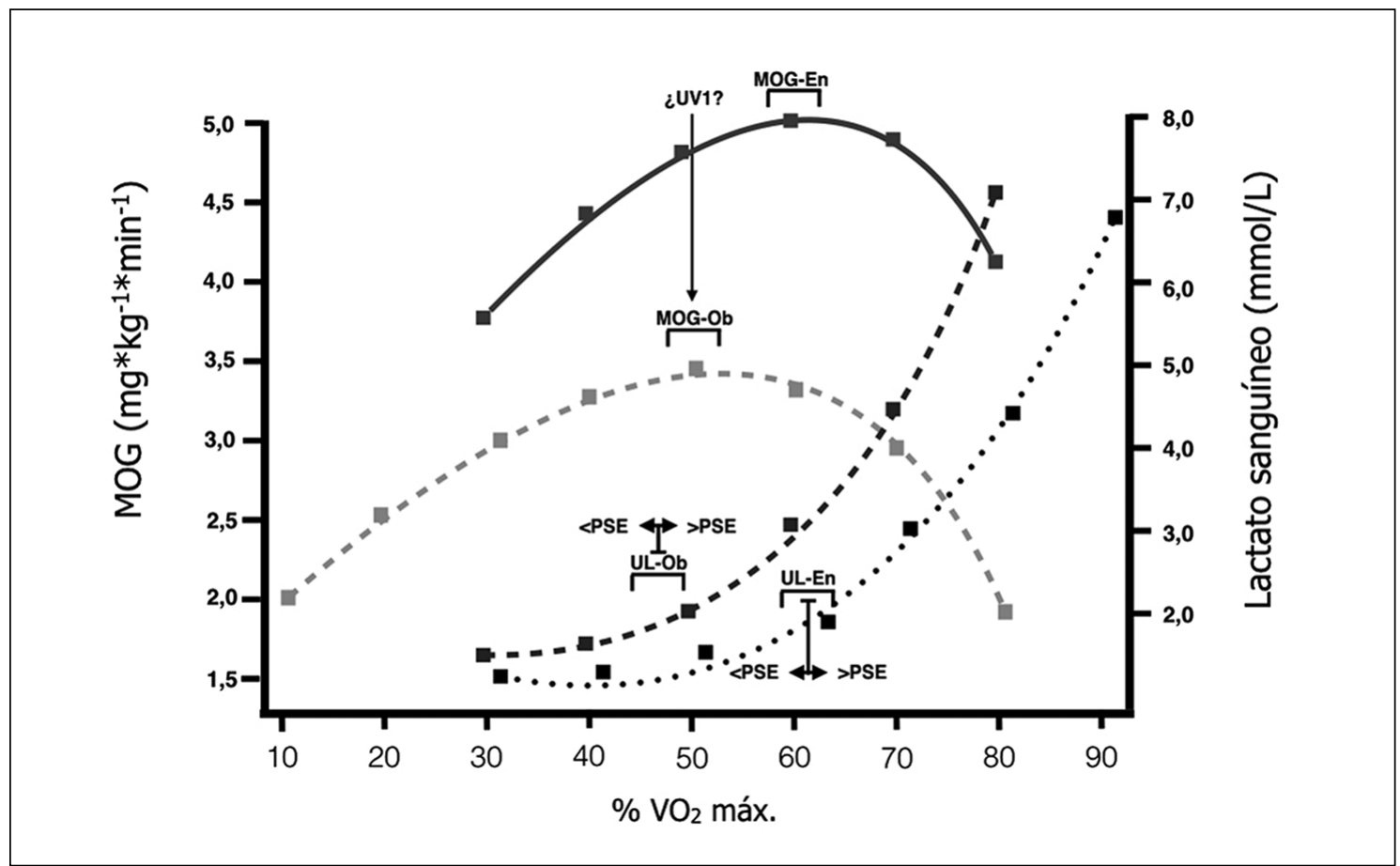

Figura 4. Esquema resumen de factores relacionados con la intensidad del ejercicio, la utilización de sustratos por el musculo esquelético y la percepción subjetiva del esfuerzo en sujetos obesos y entrenados (datos no publicados de nuestro laboratorio, de Achten y Jeukendrup ${ }^{42}$, Jeukendrup y Achten ${ }^{75}$ y Lanzi y col. ${ }^{40,76}$ ). MOG: intensidad a la cual ocurre la máxima oxidación de grasas; - - MOG-Ob: máxima oxidación de grasas en sujetos obesos; ——MOG-En: máxima oxidación de grasas en sujetos entrenados; - - UL-Ob: umbral de lactato en sujetos obesos; $\cdots$... UL-En: umbral de lactato en sujetos entrenado; PSE: percepción subjetiva del esfuerzo; UV1: umbral ventilatorio 1; mg: miligramos; kg: kilogramos; min: minuto; mmol: milimoles; L: litro; $\mathrm{VO}_{2}$ máx: consumo máximo de oxígeno.

máxima oxidación de grasas (MOG) en sujetos sanos no entrenados se encuentra entre 60 y $65 \%$ del $\mathrm{VO}_{2 \operatorname{máx}}{ }^{39}$, pero en pacientes con sobrepeso/obesidad este valor tiende a estar reducido a $45-50 \%{ }^{40}$. También se ha observado que la MOG ocurre a una misma intensidad cuando se compara entre cinta rodante y cicloergómetro, pero la tasa de oxidación de grasas (e.g., gramos $\times$ minuto $^{-1}$ ) es mayor en cinta rodante ${ }^{41}$. Se ha visto que en sujetos entrenados, la MOG está asociada a la intensidad a la que ocurre el umbral de lactato ${ }^{42}$, pero en pacientes obesos este umbral podría alcanzarse antes que la intensidad a la cual ocurre la $\mathrm{MOG}^{43}$. Esto podría ser relevante a la hora de prescribir un modelo determinado de ejercicio en pacientes con sobrepeso/obesidad, ya que esfuerzos por sobre el umbral de lactato están asociados a aumentos en la percepción subjetiva del esfuerzo ${ }^{44}$. Así, la utilización del umbral de lactato o del umbral ventilatorio1 (UV1) para prescribir ejercicio continuo podría ser una mejor estrategia para tener mayores tasas de oxidación de grasa en desmedro de los carbohidratos, ya que, como se muestra en la Figura 4, esfuerzos por debajo del umbral de lactato podrían generar una menor percepción del esfuerzo, y una menor fatiga aguda y crónica, elevando las posibilidades de generar adherencia al EF, lo cual es prioritario en las primeras etapas de intervención en el paciente con sobrepeso/ obesidad $^{45}$.

\section{Ejercicio intermitente de alta intensidad}

El ejercicio intermitente de alta intensidad (EIAI) es una modalidad de EF que implica series de ejercicio de alta/máxima intensidad y corta duración, intercaladas con series de descanso ${ }^{46}$. Inicialmente se aplicó el EF denominado all out, basado en el test de Wingate ${ }^{47}$, con respuestas 
agudas en el músculo esquelético similares a las inducidas por el ejercicio continuo ${ }^{48}$. Sin embargo, las similitudes entre estos modelos de EF respecto a las respuestas adaptativas a largo plazo son menos claras.

Si bien se considera al EIAI como un modelo específico, este corresponde a una denominación general, debido a que implica protocolos heterogéneos y con diversos efectos biológicos. Se han determinado al menos 9 variables (e.g., modalidad de ejercicio; intensidad del ejercicio; intensidad de la pausa; duración del ejercicio; duración de la pausa; número de repeticiones; número de series; duración de la recuperación entre series; tipo de recuperación entre series) que podrían modificar las características del protocolo de EF y por tanto sus efectos agudos y crónicos, tanto a nivel periférico como central ${ }^{46}$.

A pesar de lo amplio y variado de su metodología, el EIAI es un modelo de ejercicio muy popular y ampliamente utilizado en la práctica del ejercicio en diferentes poblaciones ${ }^{49}$. Se han demostrado innumerables ventajas fisiológicas y funcionales, tanto en el área deportiva, como de la salud, observándose mejoras significativas en el consumo máximo de oxígeno ( $\mathrm{VO}_{2}$ máx), elevando la capacidad física y calidad de vida ${ }^{50}$. Importantes datos clínicos muestran a este modelo de ejercicio como un método seguro y bien tolerado en pacientes en rehabilitación cardiaca, diabetes mellitus e hipertensión arterial, mejorando el $\mathrm{VO}_{2}$ máx, la función ventricular y la función endotelial ${ }^{51}$. Respecto a la regulación metabólica, se observa una importante mejora de la sensibilidad a la insulina, reduciendo los estados de hiperinsulinemia e hiperglicemia ${ }^{30}$. Nuestro grupo de investigación ha reportado mejoras en índices de resistencia a la insulina, dislipidemia y presión arterial inducidos por 8 y 12 semanas de EIAI en mujeres con sobrepeso/obesidad ${ }^{52,53}$, siendo hoy considerado una importante herramienta primaria y secundaria en el combate contra las enfermedades no transmisibles.

Sin embargo, similar al ejercicio continuo, el EIAI induce limitados efectos sobre la pérdida de peso corporal y tejido adiposo, Clark JE (2015) en un metaanálisis de 66 estudios en población adulta publicados recientemente, señala que este modelo de ejercicio genera un tamaño del efecto incierto: $0,20(-0,38,0,78)$, cuando es utilizado en forma aislado ${ }^{37,48,51}$.
En relación a la utilización de sustratos y las vías moleculares que regulan este proceso, se ha mostrado que el ejercicio intermitente de alta intensidad activa la proteína quinasa activada por AMP (AMPK), con un efecto mayor al del ejercicio continuo de intensidad baja-moderada ${ }^{58}$. También se ha observado una mayor actividad de AMPK posterior a ejercicio intermitente en comparación con el ejercicio continuo, generando una mayor inhibición de la enzima acetil-coA carboxilasa ${ }^{59}$, lo que favorecería el ingreso de los ácidos grasos a la mitocondria para su posterior oxidación. Sin embargo, debido a las altas demandas energéticas del ejercicio intermitente, la tasa a la cual se requiere energía no permite una elevada utilización de ácidos grasos como principal fuente de energía durante la sesión de ejercicio ${ }^{60}$. Además, si bien se ha observado que en algunos sujetos más entrenados, estos son capaces de oxidar más ácidos grasos durante ejercicio intermitente, la magnitud de esto y la utilización neta de este sustrato es mínima en comparación a la oxidación de carbohidratos ${ }^{61}$.

Finalmente, el tiempo en el cual los pacientes con sobrepeso/obesidad son capaces de mantener una práctica continua de EF y una vida activa son un pilar fundamental en el logro de objetivos como la pérdida de peso corporal, sin embargo, esta población en general presenta una baja adherencia a los programas de $\mathrm{EF}^{36,45,62}$. La adherencia a los protocolos de EIAI orientados a la salud pública, especialmente los denominados all out, son actualmente lugar de un amplio debate, y la adherencia a este tipo ejercicio en esta población ha sido cuestionada debido a la elevada percepción del esfuerzo, en comparación con los protocolos de ejercicio continuo de intensidad baja-moderada, lo cual podría disminuir la posibilidad de generar hábitos de vida activa en esta población ${ }^{36,45}$. No obstante, considerando los beneficios que genera el EIAI y debido a que es un modelo tiempo-eficiente, con una adecuada progresión y adaptación a las cargas de entrenamiento, podría ser complementario a utilizar en los programas de atención primaria y secundaria para pacientes con sobrepeso/obesidad.

Ejercicio de sobrecarga muscular y su valor en la disminución del peso corporal en población con sobrepeso/obesidad

La prescripción del ejercicio de sobrecarga muscular no es sencilla e involucra múltiples va- 
riables que pueden modificar sustancialmente las respuestas y adaptaciones inducidas por este. La respuesta individual a los estímulos aplicados generan una heterogeneidad amplia en los resultados de protocolos aplicados, por lo que al prescribir el ejercicio de sobrecarga muscular se deben tener en cuenta múltiples variables tales $\mathrm{como}^{63}$ : intensidad (e.g., porcentaje de una repetición máxima); número de repeticiones; número y orden de ejercicios; series por ejercicio; número de repeticiones por serie; pausas entre series y ejercicios; tipo de contracción muscular (e.g., excéntrica, concéntrica, isométrica); velocidad de ejecución del movimiento (e.g., rápida, intermedia, lenta); medio de entrenamiento (e.g., máquinas, pesos libres, elásticos, superficies inestables, equipos de suspensión); modalidad (e.g., estacionario, circuito); y la frecuencia con que serán aplicados los estímulos.

Durante la aplicación de restricción calórica en sujetos con sobrepeso/obesidad suele ocurrir una disminución importante del gasto metabólico de reposo y de la masa libre de grasa, especialmente en las primeras fases de los programas de tratamiento ${ }^{64}$. Este efecto es contrarrestado con programas de ejercicio de sobrecarga muscular, los cuales estimulan el aumento/mantención de la masa muscular y potencialmente contribuyen a un balance energético negativo ${ }^{65,66}$. Esto último podría traducirse en una disminución del tejido adiposo y del peso corporal total ${ }^{65}$, además de promover importantes adaptaciones funcionales como aumentos en la fuerza y potencia muscular ${ }^{67}$, y adaptaciones metabólicas, como un aumento en la sensibilidad a la insulina ${ }^{68,69}$.

Sin embargo, los posibles beneficios de pérdida de peso corporal de los ejercicios de sobrecarga muscular son limitados y poco alentadores. Al parecer, este modelo de ejercicio no tiene ventajas sobre otros modelos mencionados anteriormente en los resultados de pérdida de peso corporal $^{70}$. No obstante, sin considerar el impacto sobre el peso corporal total, los programas de sobrecarga muscular aplicados en pacientes con sobrepeso/ obesidad pueden inducir modificaciones significativas en la composición corporal ${ }^{71}$, la cual refleja la distribución de los diferentes compartimentos de grasa y libres de grasa en el organismo, siendo el músculo esquelético un componente esencial de este último ${ }^{72}$. La masa muscular esquelética comprende entre 40 y $50 \%$ de la masa corporal total, y entre sus principales funciones se encuentran sustentar las actividades de la vida diaria y la regulación metabólica del organismo, siendo responsable hasta $\sim 80 \%$ de la captación de glucosa inducida por insulina ${ }^{73}$. Por tanto, la mantención de la masa y función muscular protege contra enfermedades metabólicas como resistencia a la insulina, diabetes mellitus tipo 2, síndrome metabólico y dislipidemias ${ }^{74}$. Así, se ha observado que los programas de ejercicios de sobrecarga muscular realizados 3-4 veces por semana, con $2-3$ series de $6-10$ repeticiones, a una intensidad $\geq 75 \%$ de una repetición máxima y con pausas entre series de 60-90 segundos, generan mejores resultados en la mantención o aumento de la masa muscular en pacientes con sobrepeso/obesidad ${ }^{37}$.

Considerando el impacto que tiene este modelo de ejercicio sobre la masa muscular (y por consecuencia en sensibilidad a la insulina), en el gasto metabólico de reposo, en la masa corporal y en el tejido adiposo, la incorporación de ejercicios de sobrecarga en programas de tratamiento y prevención de sobrepeso/obesidad resultaría de suma importancia. Además, estos contribuyen significativamente a mejorar/mantener la función muscular, lo que favorece una adecuada realización de las actividades de la vida diaria.

\section{Conclusiones}

El ejercicio físico es piedra angular en el tratamiento de pacientes con sobrepeso/obesidad, principalmente con efectos a nivel funcional, metabólico, muscular y cardiovascular. Con dosis adecuadas de ejercicio físico también se podrían favorecer principalmente cambios en la composición corporal del paciente. Sin embargo, es importante enfatizar que la evidencia científica internacional actualizada nos muestra sólo un bajo rango de efectividad de todos los modelos de EF $(\leq 20 \%)$ en la pérdida de peso corporal total cuando esta modalidad terapéutica es aplicada en forma aislada.

Si el objetivo es la reducción del peso y la grasa corporal, la dieta o restricción calórica de los alimentos deben incluir uno varios modelos de ejercicio físico como terapia integral para maximizar las posibilidades de tratamiento efectivo.

La prescripción de ejercicio físico en pacientes con sobrepeso/obesidad debe considerar todos 
los modelos de ejercicio y sus múltiples variables biológicas y metodológicas, las cuales deben ser cuidadosamente seleccionadas en forma racional, efectiva y eficiente (e.g., de menos a más; de lo simple a lo complejo; de lo fácil a lo difícil), tomando en consideración variables interindividuales, de tolerancia y de adhesión al tratamiento, siendo esta última de especial consideración, ya que es pilar fundamental en el logro de objetivos a largo plazo en pacientes con sobrepeso/obesidad.

\section{Referencias}

1. Ladabaum U, Mannalithara A, Myer PA, Singh G. Obesity, Abdominal Obesity, Physical Activity, and Caloric Intake in U.S. Adults: 1988-2010. Am J Med 2014; 127 (8): 717-27.

2. World Health Organization. Global Status Report On Noncommunicable Diseases 2014. WHO Library Cataloguing-in-Publication Data Global; 2014. 280 p.

3. Manore MM, Brown K, Houtkooper L, Jakicic J, Peters JC, Edge MS, et al. Energy balance at a crossroads: Translating the science into action. Med Sci Sports Exerc 2014; 46 (7): 1466-73.

4. Haslam D, Sattar N, Lean M. ABC of obesity. Obesity-time to wake up. Br Med J 2006; 333: 640-2.

5. Haslam DW, James WPT. Obesity. Lancet 2005; 366: 1197-209.

6. Oster G, Thompson D, Edelsberg J, Bird AP, Colditz GA. Lifetime health and economic benefits of weight loss among obese persons. Am J Public Health 1999; 89 (10): 1536-42.

7. McArdle M, Finucane OM, Connaughton RM, McMorrow AM, Roche HM. Mechanisms of obesity-induced inflammation and insulin resistance: Insights into the emerging role of nutritional strategies. Front Endocrinol (Lausanne) 2013; 4: 1-23.

8. Denis GV, Obin MS. "Metabolically healthy obesity": Origins and implications. Mol Aspects Med 2013; 34 (1): 59-70.

9. Ruderman N, Chisholm D, Pi-Sunyer X, Schneider S. The metabolically obese, normal-weight individual revisited. Diabetes 1998; 47 (5): 699-713.

10. Wildman RP, Muntner P, Reynolds K, McGinn A, Rajpathak S, Wylie-Rosett J, et al. The Obese Without Cardiometabolic Risk Factor Clustering and the Normal Weight With Cardiometabolic Risk Factor Clustering. Arch Intern Med 2008; 168 (15): 1617-24.

11. Schinzari F, Iantorno $\mathrm{M}$, Campia $\mathrm{U}$, Mores $\mathrm{N}$, Rovella $\mathrm{V}$, Tesauro $\mathrm{M}$, et al. Vasodilator responses and endothe- lin-dependent vasoconstriction in metabolically healthy obesity and the metabolic syndrome. Am J Physiol - Endocrinol Metab 2015; 309 (9): E787-92.

12. Kramer CK, Zinman B, Retnakaran R. Are metabolically healthy overweight and obesity benign conditions?: A systematic review and meta-analysis. Ann Intern Med 2013; 159 (11): 758-69.

13. Avenell A, Brown TJ, McGee M A, Campbell MK, Grant $\mathrm{AM}$, Broom J, et al. What interventions should we add to weight reducing diets in adults with obesity? A systematic review of randomized controlled trials of adding drug therapy, exercise, behaviour therapy or combinations of these interventions. J Hum Nutr Diet 2004; 17: 293-316.

14. Curioni CC, Lourenço PM. Long-term weight loss after diet and exercise: a systematic review. Int J Obes (Lond). 2005; 29 (10): 1168-74.

15. Johns DJ, Hartmann-Boyce J, Jebb SA, Aveyard P. Diet or Exercise Interventions vs Combined Behavioral Weight Management Programs: A Systematic Review and Meta-Analysis of Direct Comparisons. J Acad Nutr Diet 2014; 114 (10): 1557-68.

16. Redman LM, Ravussin E. Caloric restriction in humans: Impact on Physiological, Psychological, and Behavioral Outcomes. Antioxid Redox Signal 2011; 14 (2): 275-87.

17. Garber CE, Blissmer B, Deschenes MR, Franklin B a, Lamonte MJ, Lee I-M, et al. American College of Sports Medicine position stand. Quantity and quality of exercise for developing and maintaining cardiorespiratory, musculoskeletal, and neuromotor fitness in apparently healthy adults: guidance for prescribing exercise. Med Sci Sports Exerc 2011; 43 (7): 1334-59.

18. Swift DL, Johannsen NM, Lavie CJ, Earnest CP, Church TS. The role of exercise and physical activity in weight loss and maintenance. Prog Cardiovasc Dis 2014; 56: 441-7.

19. Jensen MD, Ryan DH, Apovian CM, Ard JD, Comuzzie AG, Donato KA, et al. 2013 AHA/ACC/TOS guideline for the management of overweight and obesity in adults: A report of the American college of cardiology/American heart association task force on practice guidelines and the obesity society. J Am Coll Cardiol 2014; 63 (25 Part B): 2985-3023.

20. Thorogood A, Mottillo S, Shimony A, Filion KB, Joseph $\mathrm{L}$, Genest J, et al. Isolated aerobic exercise and weight loss: A systematic review and meta-analysis of randomized controlled trials. Am J Med 2011; 124 (8): 747-55.

21. Miller WC, Koceja DM, Hamilton EJ. A meta-analysis of the past 25 years of weight loss research using diet, exercise or diet plus exercise intervention. Int J Obes 1997; 21 (10): 941-7. 
22. King NA, Hopkins M, Caudwell P, Stubbs RJ, Blundell JE. Beneficial effects of exercise: shifting the focus from body weight to other markers of health. Br J Sports Med 2009; 43 (12): 924-7.

23. Jones AM, Carter H. The effect of endurance training on parameters of aerobic fitness. Sport Med. Adis International 2000; 29 (6): 373-86.

24. Keating SE, Machan EA, Connor HTO, Gerofi JA, Sainsbury A, Caterson ID, et al. Continuous Exercise but Not High Intensity Interval Training Improves Fat Distribution in Overweight Adults. J Obes 2014; 2014: 25-7.

25. Wiklund $\mathrm{P}$, Alen M, Munukka E, Cheng SM, Yu B, Pekkala S, et al. Metabolic response to 6-week aerobic exercise training and dieting in previously sedentary overweight and obese pre-menopausal women: A randomized trial. J Sport Heal Sci 2014; 3 (3): 217-24.

26. Sriwijitkamol A, Coletta DK, Wajcberg E, Balbontin GB, Reyna SM, Barrientes J, et al. Effect of acute exercise on AMPK signaling in skeletal muscle of subjects with type 2 diabetes: a time-course and dose-response study. Diabetes 2007; 56 (3): 836-48.

27. Bradley H, Shaw CS, Worthington PL, Shepherd SO, Cocks M, Wagenmakers AJM. Quantitative immunofluorescence microscopy of subcellular GLUT4 distribution in human skeletal muscle: effects of endurance and sprint interval training. Physiol Rep 2014; 2 (7): $1-16$.

28. Kennedy J, Hirshman M, Gervino E, Ocel J, Forse RA, Hoenig S, et al. Acute Exercise Induces GLUT4 Translocation in Skeletal Muscle of Normal Human Subjects and Subjects With Type 2 Diabetes. Diabetes 1999; 48: $1-6$.

29. Houmard JA, Tanner CJ, Slentz CA, Duscha BD, McCartney JS, Kraus WE. Effect of the volume and intensity of exercise training on insulin sensitivity. J Appl Physiol 2004; 96 (1): 101-6.

30. Little JP, Jung ME, Wright AE, Wright W, Manders RJF. Effects of high-intensity interval exercise versus continuous moderate-intensity exercise on postprandial glycemic control assessed by continuous glucose monitoring in obese adults. Appl Physiol Nutr Metab 2014; 39 (7): 835-41.

31. Nicklas BJ, Katzel LI, Busby-Whitehead J, Goldberg AP. Increases in high-density lipoprotein cholesterol with endurance exercise training are blunted in obese compared with lean men. Metabolism 1997; 46 (18): 556-61.

32. Fiuza-Luces C, Garatachea N, Berger NA, Lucia A. Exercise is the real polypill. Physiology (Bethesda) 2013; 28 (5): 330-58.
33. Gibala MJ. High-intensity Interval Training: A Time-efficient Strategy for Health Promotion? Curr Sports Med Rep 2007; 6: 211-3.

34. Gillen JB, Gibala MJ. Is high-intensity interval training a time-efficient exercise strategy to improve health and fitness? Appl Physiol Nutr Metab 2014; 39 (3): 409-12.

35. Hainer V, Toplak H, Stich V. Fat or fit: what is more important? Diabetes Care 2009; 32 Suppl 2: s392-7.

36. Saanijoki T, Nummenmaa L, Eskelinen J-J, Savolainen AM, Vahlberg T, Kalliokoski KK, et al. Affective Responses to Repeated Sessions of High-Intensity Interval Training. Med Sci Sport Exerc 2015; 47 (12): 1-8.

37. Clark JE. Diet, exercise or diet with exercise: comparing the effectiveness of treatment options for weight-loss and changes in fitness for adults (18-65 years old) who are overfat, or obese; systematic review and meta-analysis. J Diabetes Metab Disord 2015; 14 (31): 1-28.

38. Washburn RA, Szabo AN, Lambourne K, Willis EA, Ptomey LT, Honas JJ, et al. Does the Method of Weight Loss Effect Long-Term Changes in Weight, Body Composition or Chronic Disease Risk Factors in Overweight or Obese Adults? A Systematic Review. PLoS One 2014; 9 (10): e109849.

39. Jeukendrup AE. Regulation of fat metabolism in skeletal muscle. Ann N Y Acad Sci 2002; 967: 217-35.

40. Lanzi S, Codecasa F, Cornacchia M, Maestrini S, Salvadori A, Brunani A, et al. Fat oxidation, hormonal and plasma metabolite kinetics during a submaximal incremental test in lean and obese adults. PLoS One 2014; 9 (2): e88707.

41. Achten J, Jeukendrup AE. Optimizing fat oxidation through exercise and diet. Nutrition 2004; 20 (7-8): 716-27.

42. Achten J, Jeukendrup AE. Relation between Plasma Lactate Concentration and Fat Oxidation Rates over a Wide Range of Exercise Intensities. Int J Sports Med 2004; 25 (1): 32-7.

43. Bircher S, Knechtle B. Relationship between fat oxidation and lactate threshold in athletes and obese women and men. J Sports Sci Med 2004; 3: 174-81.

44. Demello JJ, Cureton KJ, Boineau RE, Singh MM. Ratings of perceived exertion at the lactate threshold in trained and untrained men and women. Med Sci Sports Exerc 1987; 19 (4): 354-62.

45. Biddle SJH, Batterham AM. High-intensity interval exercise training for public health: a big HIT or shall we HIT it on the head? Int J Behav Nutr Phys Act 2015; 12 (1): 95 .

46. Buchheit M, Laursen PB. High-intensity interval training, solutions to the programming puzzle: Part I: 
cardiopulmonary emphasis. Sports Med 2013; 43 (5): 313-38.

47. Gibala M. Molecular responses to high-intensity interval exercise. Appl Physiol Nutr Metab 2009; 34 (3): 428-32.

48. Gibala MJ, Little JP, Macdonald MJ, Hawley JA. Physiological adaptations to low-volume, high-intensity interval training in health and disease. J Physiol 2012; 590: 1077-84.

49. Logan GRM, Harris N, Duncan S, Schofield G. A review of adolescent high-intensity interval training. Sports Medicine 2014; 44: 1071-85.

50. Milanovi Z, Sporiš G, Weston M. Effectiveness of High-Intensity Interval Training (HIT) and Continuous Endurance Training for VO2max Improvements: A Systematic Review and Meta-Analysis of Controlled Trials. Sport Med 2015; 45: 1469-81.

51. Guiraud T, Nigam A, Gremeaux V, Meyer P, Juneau M, Bosquet L. High-intensity interval training in cardiac rehabilitation. Sports Med 2012; 42 (7): 587-605.

52. Álvarez L. C, Ramírez-Campillo R, Flores OM, Henríquez-Olguín C, Campos JC, Carrasco V, et al. Respuestas metabólicas inducidas por ejercicio físico de alta intensidad en mujeres sedentarias con glicemia basal alterada e hipercolesterolemia. Rev Med Chile 2013; 141: 1293-9.

53. Álvarez C, Ramírez-Campillo R, Henríquez-Olguín C, Castro-Sepúlveda M, Carrasco V, Martínez C. ¿Pueden ocho semanas de ejercicio físico combinado normalizar marcadores metabólicos de sujetos hiperglicémicos y dislipidémicos? Rev Med Chile 2014; 142: 458-66.

54. Boutcher SH. High-Intensity Intermittent Exercise and Fat Loss. J Obes 2011; 2011: 868305: 1-10.

55. Gillen JB, Gibala MJ. Is high-intensity interval training a time-efficient exercise strategy to improve health and fitness? Appl Physiol Nutr Metab 2014; 39 (3): 409-12.

56. Gillen JB, Percival ME, Ludzki A, Tarnopolsky MA, Gibala MJ. Interval Training in the Fed or Fasted State Improves Body Composition and Muscle Oxidative Capacity in Overweight Women. Obesity 2013; 21 (11): 2249-55.

57. Whyte LJ, Gill JMR, Cathcart AJ. Effect of 2 weeks of sprint interval training on health-related outcomes in sedentary overweight / obese men. Metab Clin Exp 2010; 59 (10): 1421-8.

58. Birk JB, Wojtaszewski JFP. Predominant alpha2/beta2/ gamma3 AMPK activation during exercise in human skeletal muscle. J Physiol 2006; 577: 1021-32.

59. Kristensen DE, Albers PH, Prats C, Baba O, Birk JB, Wojtaszewski JFP. Human muscle fibre type-specific re- gulation of AMPK and downstream targets by exercise. J Physiol 2015; 593 (8): 2053-69.

60. Yoshida Y, Jain SS, McFarlan JT, Snook LA, Chabowski A, Bonen A. Exercise- and training-induced upregulation of skeletal muscle fatty acid oxidation are not solely dependent on mitochondrial machinery and biogenesis. J Physiol 2013; 591 (Pt 18): 4415-26.

61. Hetlelid KJ, Plews DJ, Herold E, Laursen PB, Seiler S. Rethinking the role of fat oxidation: substrate utilisation during high-intensity interval training in well-trained and recreationally trained runners. BMJ Open Sport Exerc Med 2015; 1 (1): e000047.

62. Colley RC, Hills AP, O’Moore-Sullivan TM, Hickman IJ, Prins JB, Byrne NM. Variability in adherence to an unsupervised exercise prescription in obese women. Int J Obes (Lond) 2008; 32 (5): 837-44.

63. Ratamess NA. ACSM's Foundations of Strength Training and Conditioning. Lupash E, editor. Vasa. Wolters Kluwer; 2012. 514 p.

64. Redman LM, Heilbronn LK, Martin CK, de Jonge L, Williamson DA, Delany JP, et al. Metabolic and Behavioral Compensations in Response to Caloric Restriction: Implications for the Maintenance of Weight Loss. PLoS One 2009; 4 (2): e4377.

65. Drenowatz C, Hand GA, Sagner M, Shook RP, Burgess S, Blair SN. The Prospective Association Between Different Types of Exercise and Body Composition. Med Sci Sport Exerc 2015; 47 (12): 2535-41.

66. Strasser B, Arvandi M, Siebert U. Resistance training, visceral obesity and inflammatory response: A review of the evidence. Obes Rev 2012; 13 (7): 578-91.

67. American College of Sports Medicine. American College of Sports Medicine position stand. Progression models in resistance training for healthy adults. Med Sci Sports Exerc 2009; 41 (3): 687-708.

68. Abd El-Kader SM. Aerobic versus resistance exercise training in modulation of insulin resistance, adipocytokines and inflammatory cytokine levels in obese type 2 diabetic patients. J Adv Res Cairo University 2011; 2 (2): 179-83.

69. Rice B, Janssen I, Hudson R, Ross R. Effects of Aerobic or Resistance Exercise and/or Diet on Glucose Tolerance and Plasma Insulin Levels in Obese Men. Diabetes Care 1999; 22 (5): 684-91.

70. Jakicic JM, Otto A. Physical activity considerations for the treatment and prevention of obesity. Int J Obes Am J Clin Nutr 2005; 82 (suppl): 226-9.

71. Miller CT. Effect of Diet versus Diet and Exercise on Weight Loss and Body Composition in Class II and III Obesity: A Systematic Review. J Diabetes Metab 2013; 4 (6): 2-7.

72. Switzer NJ, Mangat HS, Karmali S. Current trends in 
obesity: body composition assessment, weight regulation, and emerging techniques in managing severe obesity. J Interv Gastroenterol 2013; 3 (1): 34-6.

73. DeFronzo RA, Jacot E, Jequier E, Maeder E, Wahren J, Felber JP. The effect of insulin on the disposal of intravenous glucose. Results from indirect calorimetry and hepatic and femoral venous catheterization. Diabetes 1981; 30 (12): 1000-7.

74. Brook MS, Wilkinson DJ, Phillips BE, Pérez-Schindler J, Philp A, Smith K, et al. Skeletal muscle homeostasis and plasticity in youth and ageing: impact of nutrition and exercise. Acta Physiol 2015; 1-27.

75. Jeukendrup A, Achten J. Fatmax: A new concept to optimize fat oxidation during exercise? Eur J Sport Sci 2001; 1 (5): 1-5.

76. Lanzi S, Codecasa F, Cornacchia M, Maestrini S, Capodaglio P, Brunani A, et al. Short-term HIIT and Fat max training increase aerobic and metabolic fitness in men with class II and III obesity. Obesity 2015; 23 (10): 1987-94. 